\title{
Low Dose-Olanzapine-Induced Neuroleptic Malignant Syndrome in Adolescent, and Literature Review
}

\author{
Sulhi A. Alfakeh, MBBS, FRCPC \\ Department of Internal Medicine, Faculty of Medicine, King Abdulaziz University \\ Jeddah, Saudi Arabia
}

\section{Correspondence}

Dr. Sulhi A. Alfakeh

Department of Internal Medicine

Faculty of Medicine, King Abdulaziz University

P.O. Box 80216, Jeddah 21589, Saudi Arabia

e.M: sulhi.alfakeh@hotmail.com

\section{Submission: 02 Sept. 2018}

Accepted: 10 Sept. 2018

\section{Citation}

Alfakeh SA. Low dose-olanzapine-induced neuroleptic malignant syndrome in adolescent, and literature review. JKAU Med Sci 2018; 25 (2): 43-49. DOI: 10.4197/Med. 25.2.5

Copyright: $\odot$ The Author(s), YEAR. Publisher. The Journal of King Abdualziz University - Medical Sciences is an Official Publication of "King Abdulaziz University". It is an open-access article distributed under the terms of the Creative Commons Attribution Non-Commercial License, which permit unrestricted non-commercial use, distribution, and reproduction in any medium, provided the original work is properly cited.

\begin{abstract}
Neuroleptic malignant syndrome is a rare but potentially lethal condition associated with neuroleptic medications. It is characterized by high temperature, extrapyramidal symptoms, autonomic variability, and changes in consciousness. Although olanzapine is an atypical antipsychotic, which exhibits more affinity to serotonin receptors than to dopamine receptors, atypical antipsychotics (like olanzapine) can cause "typical and atypical" forms of neuroleptic malignant syndrome. Previous reports of neuroleptic malignant syndrome associated with olanzapine have described patients who had been previously treated with other antipsychotic medications, patients who had previous incidents of neuroleptic malignant syndrome caused by other neuroleptics, or patients treated with olanzapine and another neuroleptic. This article describes a case of neuroleptic malignant syndrome linked to olanzapine in a male adolescent, 17-year old, who had not previously been treated with antipsychotic medications. He presented in an agitated state, with acute onset of fever, catatonia for 15 days, and severe negativism, refusal to eat, mutism, and brief episodes of abrupt psychomotor agitation and aggression toward his family.
\end{abstract}

\section{Keywords}

Neuroleptic Malignant Syndrome; Olanzapine; Adolescent

\section{Introduction}

$N$ euroleptic malignant syndrome (NMS) is rare and potentially lethal, normally treated with antipsychotics ${ }^{[1]}$. The diagnosis is established on clinical criteria (DSM-5), laboratory data and exclusion of other possible causes ${ }^{[2]}$. The incidence of NMS is within the range of $0.2-3.23 \%$ of patients treated with neuroleptics ${ }^{[3]}$. It is primarily caused by dopamine (D2) receptors blockage in the nigrostriatal tract, mesocortical pathway, and hypothalamic nuclei ${ }^{[1]}$. Atypical neuroleptics are a novel group of antipsychotic medications with both dopaminergic and serotonergic neurochemical effects, with considerably reduced potential for extrapyramidal side effects, and less severe adverse effects than traditional agents ${ }^{[3,4]}$. Neuroleptic malignant syndrome is usually seen in treatment with high potency typical neuroleptics and very infrequently with atypical antipsychotics. The reduced extrapyramidal effects of atypical neuroleptics minimize the muscle rigidity as well as the temperature dysregulation thought to be essential in the pathophysiology of NMS. 
This report describes a case of neuroleptic malignant syndrome associated with olanzapine in a 17-year old male adolescent who had not previously been exposed to other neuroleptics.

\section{Case Report}

A 17-year-old African adolescent brought in by his family to the emergency department in an agitated state with an acute onset of fever, headache, nausea and vomiting three times during the preceding day. He presented with a catatonic status for 15 days, characterized by intense negativism, a refusal to eat, mutism, brief episodes of abrupt psychomotor agitation, and aggressive behaviour against his family. He was well until 2 weeks prior to presentation when he started talking about paradise and a need to be more religious. He was not hearing voices, had no delusions, and had no prior medical or psychiatric illness as reported by his father. His father took him to a private hospital where a diagnosis of schizophrenia was suggested, and the patient was started on clonazepam $2 \mathrm{mg}$ three times per day, on which the patient became very sedated. His outpatient psychiatrist decreased clonazepam dose to $1 \mathrm{mg} 3$ times per day and added olanzapine $5 \mathrm{mg}$ twice a day. Two weeks later, he became very sedated and dysarthric, was not eating, and had rigidity. There was no current or past history of alcohol or illicit drug use, or head trauma. His family stopped all medications and brought him to the emergency department. On arrival, his vital signs were as follows: a temperature of $38.4^{\circ} \mathrm{C}$, blood pressure of $135 / 80 \mathrm{~mm} \mathrm{Hg}$, a pulse of 113 , respiratory rate of 22 , and oxygen saturation of $96 \%$ on room air. The patient was pale, cachectic, and dehydrated. Further examination revealed most notably marked neck stiffness, dysarthria, positive Brudzinski and Kernig signs, and rigidity of extremities with brisk reflexes of the upper and lower limbs. The rest of his physical examination was normal. The patient was admitted to the intensive care unit with working differential diagnoses of drug overdose, drug side effects, recreational drug toxicity, infection including tropical diseases, occult central nervous system (CNS) pathology, NMS, and toxin exposure. Laboratory data showed a white blood cell (WBC) count of 17,800 cells/ $\mathrm{mm}^{3}$ with $70 \%$ neutrophils, one band, platelets of 320,000 platelets $/ \mathrm{mm}^{3}$, creatine kinase (CK), $470 \mathrm{U} / \mathrm{L}$ (normal: 55-170), lactate dehydrogenase (LDH), 569 U/L (normal: 100-190) amylase, 24 U/L (normal: 25115), sodium ( $\mathrm{Na}), 128 \mathrm{mmol} / \mathrm{L}$, chloride, $89 \mathrm{mmol} / \mathrm{L}$, aspartate aminotransferase (AST), $222 \mathrm{U} / \mathrm{L}$ (normal: 1537), alanine aminotransferase (ALT), $112 \mathrm{U} / \mathrm{L}$ (normal:
30-65), normal total protein, albumin, and alkaline phosphatase (AIP), and erythrocyte sedimentation rate (ESR), $09 \mathrm{~mm} / \mathrm{H}$ (normal: 1-20). Cerebral spinal fluid (CSF) was normal with glucose of $4.0 \mathrm{mmol} / \mathrm{L}$ (normal: 2.3-4.1) and protein of $0.28 \mathrm{~g} / \mathrm{L}$. Computed tomography of the head was normal. Urine toxicology screen for illicit drugs was negative; alcohol, salicylate, and acetaminophen levels were negative. Based on these findings and the patient's symptoms, the treating team made a presumptive diagnosis of meningoencephalitis. He was started on ceftriaxone 2 gm intravenously every 12 hours and vancomycin $1 \mathrm{gm}$ intravenously every 12 hours. Dexamethasone $8 \mathrm{mg}$ intravenously every 6 hours and acyclovir $800 \mathrm{mg}$ intravenously every 8 hours were added later. Blood and CSF cultures were negative. Magnetic resonance imaging of the brain was negative, and electroencephalogram was normal. Initially, he received no specific pharmacotherapy for NMS. Following psychiatric consultation, the patient was started on dantrolene $25 \mathrm{mg}$ twice a day and trihexyphenidyl. Two days later, he became afebrile and began to have periods of consciousness. The abnormal laboratory values normalized. The patient gained full consciousness, and he was transferred to the general ward.

\section{Discussion}

The above case provides an opportunity to review the current literature regarding the incidence, clinical presentation, and management of NMS in adolescents. Neuroleptic malignant syndrome has been described since 1960 by French clinicians in a study of haloperidol and subsequently named the "syndrome malin des neuroleptiques". This nomenclature struggled in translation to the neuroleptic malignant syndrome, and the term has sustained in the front of continued debate ${ }^{[5,6]}$. Neuroleptic malignant syndrome is the most serious of acute neurological adverse effects caused by neuroleptics, and characterized by hyperthermia, changed consciousness (e.g., confusion, catatonia, and stupor), lead-pipe rigidity, autonomic dysfunction, markers of muscle injury (e.g., high creatine kinase levels), leukocytosis $(10,000$ to 40,000 cells/mm3 with a left shift), tremors, diaphoresis, hyper-salivation, and extrapyramidal signs that may precede $\mathrm{NMS}^{[3-5,7-}$ 10]. Usually, extrapyramidal symptoms occur before autonomic symptoms do ${ }^{[11]}$.

Olanzapine is a thienobenzodiazepine atypical antipsychotic related to clozapine that acts on dopaminergic, serotoninergic, histaminergic, and 
muscarinic receptors ${ }^{[7]}$. In an effort to preserve the novel therapeutic profile of clozapine while reducing the danger of agranulocytosis, researchers developed a close chemical analog of clozapine named "olanzapine". In premarketing studies of more than 2,500 patients who used olanzapine before its introduction in 1996, no cases of NMS were published ${ }^{[8]}$. Neuroleptic malignant syndrome frequently develops within seven days after the start of treatment with antipsychotics ${ }^{[9,11,12]}$. In our patient, symptoms of NMS started within 48 hours after initiation of olanzapine. The duration of described symptoms has ranged from 1 to 44 days with an average continuation of symptoms of 1 to 10 days ${ }^{[11]}$. This incident was slightly unusual, owing to the unexpected onset of psychotic symptoms followed by the almost sudden development of severe NMS upon brief, low doses and first exposure to atypical antipsychotic medications. It is uncommon for NMS to develop after 30 days of treatment unless the dose increases or another antipsychotic drug is added ${ }^{[7]}$. Atypical presentations of NMS present a diagnostic difficulty as hyperthermia, autonomic instability, muscle rigidity, and elevated creatine kinase may be absent, evolve gradually over several days, or present in ranging levels of severity, leading to the premature exclusion of a diagnosis of NMS, an impairment or significant delay in diagnosis and management, or a prolonged course of NMS and raised risks of complications, long-term consequences, and possibly death ${ }^{[7,9]}$. Hence, NMS can be viewed as a spectrum illness. Atypical antipsychotics (like olanzapine) were observed more likely than typical antipsychotics to be connected with NMS presentation that lacked one of the main symptoms, like hyperthermia ${ }^{[7]}$.

The pathophysiological mechanism of NMS is not entirely known and is believed to be caused by central and peripheral dopaminergic blockage that manifests in temperature elevations, muscle rigidity, and hypermetabolism $^{[1,12]}$. The observation supports this hypothesis that dopamine D2 antagonists cause NMS, and dopamine agonists are often useful in treating $\mathrm{NMS}^{[7]}$.

Neuroleptic malignant syndrome has been described with atypical antipsychotic medications, which are considered to have much less dopamine blockade than typical antipsychotics, as well as additional receptor effects, other factors are implicated. Lately, several views have expressed that NMS is not caused by dopamine block alone, and other receptors may be involved, such as norepinephrine, serotonin, and $\mathrm{GABA}^{[1]}$. First, the central thermoregulation is regulated by interactions between the noradrenergic, serotoninergic, dopaminergic, and cholinergic pathways, it is questionable that dopamine blockade of neuroleptic drugs is the only cause of $\mathrm{NMS}^{[11]}$. Second, the primary cause of hyperthermia is thought to be caused by the dopaminergic blockade that affects serotoninergic pathways in the hypothalamus. Third, the lack of core symptoms of NMS like hyperthermia or muscle rigidity in atypical cases of NMS supports the probability of different pathophysiological explanations ${ }^{[11]}$. For example, one research group reported significantly higher circulatory levels of epinephrine and serotonin in patients with NMS during the acute phase compared with levels obtained after recovery ${ }^{[8]}$.

Therefore, these findings suggest that dysfunction in these neurotransmitter systems, in addition to dopamine, may be involved in the pathogenesis of $\mathrm{NMS}^{[5-14]}$. Common risk factors for NMS appear to be linked with a number of pharmacologic factors, in addition to many clinical and systemic risk factors

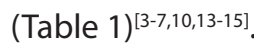

Even with immediate identification, NMS causes high rates of morbidity and mortality ${ }^{[8]}$. The reported mortality rate is estimated at approximately 10\%, and it is usually self-limited ${ }^{[6,9]}$, Mortality is lower in NMS caused by second-generation antipsychotics than in NMS linked to first- generation agents. The mortality rate may simply reflect physicians' awareness and ensuing early treatment. Complications from untreated hyperthermia, myoglobinuria, and autonomic dysregulation cause most of the untoward consequences of the syndrome, and the clinical

Table 1. Risk factors for neuroleptic malignant syndrome.

\begin{tabular}{|l|l|}
\hline 1 & Prior history of Neuroleptic Malignant Syndrome \\
\hline 2 & $\begin{array}{l}\text { Prior neuroleptic exposure (esp. the use of older, high-potency } \\
\text { typical neuroleptics) }\end{array}$ \\
\hline 3 & Rapid increase in dose of drug (rapid titration) \\
\hline 4 & Dehydration \\
\hline 5 & Young age \\
\hline 6 & Male gender \\
\hline 7 & Recent trauma \\
\hline 8 & Agitation \\
\hline 9 & Physical restraint \\
\hline 10 & Iron deficiency \\
\hline 11 & Pre-existing central dopamine abnormalities \\
\hline 12 & Mental retardation \\
\hline 13 & Adjunctive use of lithium \\
\hline 14 & Stress or exhaustion \\
\hline
\end{tabular}


outcome of those who recover from the syndrome is generally good ${ }^{[3]}$. If unrecognized or untreated, severe complications, like aspiration pneumonia secondary to obtundation or dysphagia, acute renal failure, cardiac arrest and fatal arrhythmias, pulmonary embolism secondary to rigidity, disseminated intravascular coagulation and thrombocytopenia, or death may result ${ }^{[7]}$. The most vital factor for prognosticating death in patients with neuroleptic malignant syndrome $(50 \%$ correspondence rate) is the new onset or worsening of renal failure ${ }^{[1,11,12]}$.

Treatment of NMS starts with immediate identification and withdrawal of antipsychotic medications. Patients with NMS require supportive care, and several medication interventions, like amantadine, bromocriptine, and dantrolene ${ }^{[7]}$. First, all neuroleptics must be discontinued immediately. This poses a problem with the depot preparations and generally lengthens the course of treatment for these patients. Second, patients require supportive care, often in an intensive care setting, which includes control of fever (antipyretics and cooling sheets), aspiration precautions, and deep venous thrombosis prevention; nutritional support needs to be addressed ${ }^{[12]}$. Supportive care also includes support of respiratory, cardiovascular, and renal functions. Control of blood pressure and cardiovascular and autonomic functions may require medication and cardiologic consultation. Respiratory function may require mechanical ventilation for airway protection. Treatment of dehydration, myoglobinuria, and renal and electrolyte abnormalities requires monitoring of blood chemistries and intravenous fluids. Muscle rigidity leading to ischemia and hyperthermia combine to precipitate myoglobinuria, which increases the risk of renal failure. Third, there are pharmacological treatments for NMS and for supportive care. Bromocriptine, a dopamine agonist (thought to work to reverse the dopamine receptor blockade of neuroleptics), can be administered at a dose between 2.5 to $10 \mathrm{mg}$ every 6-8 hours as required to resolve the symptoms of NMS. The possible side effects of bromocriptine include hypotension and the potential aggravation of psychosis. In cases of severe hypertension, sodium nitroprusside infusion decreases blood pressure and reduces core body temperature by increasing heat radiation from the $\mathrm{skin}^{[12]}$. Dantrolene, which is used as a muscle relaxant in malignant hyperthermia associated with anesthesia, has been used in NMS to decrease the body temperature ${ }^{[12]}$ and muscle rigidity ${ }^{[5]}$ by inhibiting calcium release from the muscle sarcoplasmic reticulum. Dantrolene is given intravenously at an initial dose of $2 \mathrm{mg} / \mathrm{kg}$ followed by doses of $1 \mathrm{mg} / \mathrm{kg}$ repeated every 6 hours as needed to a maximum of $10 \mathrm{mg} / \mathrm{kg}$ per day. The reduction of the core body temperature should be evident within 10 minutes after dantrolene administration. Caution should be used in administrating dantrolene because of its possible hepatotoxicity. Drug interactions with calcium channel blockers like verapamil may cause severe hyperkalemia, myocardial depression, heart block, or death because both medications work through the calcium mechanisms of muscle contraction ${ }^{[6,12]}$. Dantrolene and bromocriptine can be administered at the same time without any severe side effect.

Supportive medications also include the benzodiazepines (esp. the use of lorazepam) that can be used for the treatment of agitation ${ }^{[5]}$. Anticholinergics may worsen the CNS effects of delirium, confusion, and hyperthermia and are generally not recommended ${ }^{[3,12]}$. Electroconvulsive therapy (ECT) is a potential intervention that can be fast acting for NMS. Electroconvulsive therapy is not itself a possible risk factor for NMS, especially in cases of NMS with catatonic-like symptoms $s^{[5,9,12]}$.

Pharmacologic treatments of NMS can be extended for 1 to 3 weeks to prevent potential recurrence of symptoms ${ }^{[12,13]}$. After the first occurrence of NMS, the following question often arises: can the patient receive another neuroleptic medication from the same therapeutic group again? Reinstitution of equipotent neuroleptic results in approximately an $80 \%$ recurrence rate of NMS. Nevertheless, only $10 \%$ of patients will have NMS if a lower potency antipsychotic drug or a lower dose of the initiating antipsychotic is used $^{[11]}$. A lower dose of a low-potency antipsychotic can be started 2 to 3 weeks after an episode of NMS as long as the patient is carefully monitored and followed ${ }^{[5,8,12]}$. Our patient's psychotic symptoms did not return after the resolution of NMS episode, so the challenge to restart antipsychotic medication was not a pressing concern. Although successfully restarting the same antipsychotic medication has been described, the literature also recommends that using a different, lower-potency agent is safer ${ }^{[5,8]}$. Moreover, the recurrence of NMS with olanzapine treatment has been observed ${ }^{[4]}$.

During recent years, "serotonin syndrome" (SS) has been reported as a result of serotoninergic hyperstimulation due to administration of antidepressant medications. The common clinical 
characteristics of serotonin syndrome are changes in restlessness, mental status, hyperreflexia, and myoclonus. The overlap of the clinical symptoms of olanzapine-induced NMS and SS implies associations in their underlying pathophysiology ${ }^{[1]}$. Additionally, the challenges of distinguishing between NMS and SS have been well identified.

To our knowledge, the diagnosis of schizophrenia was given to our patient, as reported by his father, by his outpatient psychiatrist. However, we do not have a medical report to document such. Retrospective history provided by his family and our psychiatric assessment did not support such a diagnosis. The neuroleptic malignant syndrome can occur with atypical antipsychotics like olanzapine especially in the presence of risk factors. We should pay attention to this rare but potentially fatal complication. Comprehensive psychiatric assessment before initiating psychotropic medication is essential to determine the nature of the psychiatric illness and whether antipsychotic medication is an appropriate course of action, as well as to provide close monitoring regarding use and dosing of antipsychotic medications.

\section{Conclusion}

Appropriate caution is indicated when using antipsychotic medications, particularly because of their widespread usage for psychotic and nonpsychotic disorders in the child and adolescent population. Despite the fact that antipsychotic agents can be effective, the possible hazards they pose need to be identified and recognized. Although NMS is a rare side effect due to olanzapine, it can be life-threatening, and require prompt recognition and management. Appropriate and close monitoring for children and adolescents when antipsychotics are prescribed is highly suggested. Close communication between medical and psychiatric services is crucial in cases of NMS.

\section{Conflict of Interest}

The author has no conflict of interest.

\section{Disclosure}

The author did not receive any type of commercial support either in forms of compensation or financial for this case report. The author has no financial interest in any of the products or devices, or drugs mentioned in this article.

\section{Ethical Approval}

Obtained.

\section{References}

[1] Kontaxakis VP, Havaki-Kontaxaki BJ, Christodoulou NG, Paplos KG, Christodoulou GN. Olanzapine-associated neuroleptic malignant syndrome: Is there an overlap with the serotonin syndrome? Ann Gen Hosp Psychiatry 2003; 2(1): 10 .

[2] The Diagnostic and Statistical Manual of Mental Disorders, Fifth Edition, American Psychiatric Association, 2013

[3] Hall KL, Taylor WH, Ware MR. Neuroleptic malignant syndrome due to olanzapine. Psychopharmacol Bull 2001; 35(3): 49-54.

[4] Marshall PB, MellmanTA, Nguyen SX. Neuroleptic malignant syndrome with the addition of aripiprazole to olanzapine. Am J Psychiatry 2008; 165(11): 1488-1489.

[5] Philibert RA, Adam LA, Frank FM, Carney-Doebbeling C. Olanzapine usage associated with neuroleptic malignant syndrome. Psychosomatics 2001; 42(6): 528-529.

[6] Berry N, Pradhan S, Sagar R, Gupta SK. Neuroleptic malignant syndrome in an adolescent receiving olanzapine-lithium combination therapy. Pharmacotherapy 2003; 23(2): 255259

[7] López-Torres E, Cobo I, Lucena MI. [Olanzapine-associated neuroleptic malignant syndrome. A case report and favorable response to risperidone]. Actas Esp Psiquiatr 2007; 35(2): 145-146.

[8] Picard LS, Lindsay S, Strawn JR, Kaneria RM, Patel NC, Keck PE Jr. Atypical neuroleptic malignant syndrome: diagnostic controversies and considerations. Pharmacotherapy 2008; 28(4): 530-535

[9] Onose M, Kawanishi C, Onishi H, Yamada T, Itoh M, Kosaka K, Taguchi J, Fujisawa S, Kanamori H. Neuroleptic malignant syndrome following BMT. Bone Marrow Transplant 2002; 29(9): 803-804.

[10] Mishra B, Mishra B, Sahoo S, Arora M, Khess CR. Atypicality in presentation of neuroleptic malignant syndrome caused by olanzapine. Indian J Med Sci 2007; 61(10): 570-573.

[11] Chandran GJ, Mikler JR, Keegan DL. Neuroleptic malignant syndrome: case report and discussion. CMAJ 2003; 169(5): 439-442.

[12] Hall RC, Appleby B, Hall RC. Atypical neuroleptic malignant syndrome presenting as fever of unknown origin in the elderly. South Med J 2005; 98(1): 114-117.

[13] Gheorghiu S, Knobler HY, Drumer D. Recurrence of neuroleptic malignant syndrome with olanzapine treatment. Am J Psychiatry 1999; 156(11): 1836.

[14] Suh H, Bronson B, Martin R. Neuroleptic malignant syndrome and low-dose olanzapine. Am J Psychiatry 2003; 160(4): 796. 
Low Dose-Olanzapine-Induced Neuroleptic Malignant Syndrome in Adolescent, and Literature Review S.A. Alfakeh

[15] Corallo CE, Ernest D. Atypical neuroleptic malignant syndrome with long-term clozapine. Crit Care Resusc 2007; 9(4): $338-340$ 


\section{جرعة منخفضة من عقار الأولانزيبين تسببت في حدوث المتلازمة الخبيثة

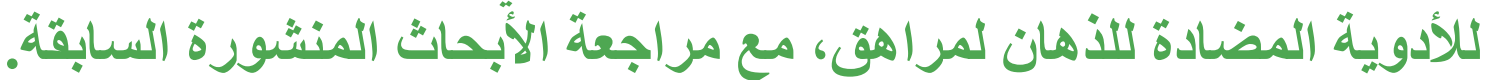

$$
\begin{aligned}
& \text { صلحي علي الفقية }
\end{aligned}
$$

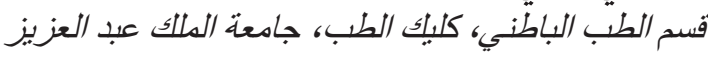

$$
\begin{aligned}
& \text { جدة - المملكة العربية السعودية العية }
\end{aligned}
$$

المستخلص. تعتبر المتلازمة الخبيثة للأدوية المضادة للذهان حالة نادرة ولكنها قد تكون مميتة حيث ترتبط مباشرة بالأدوية

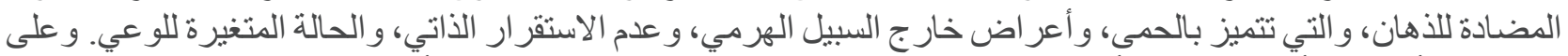

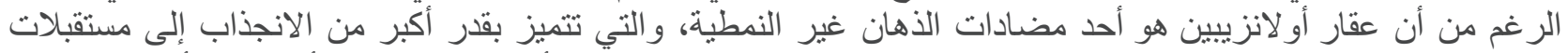

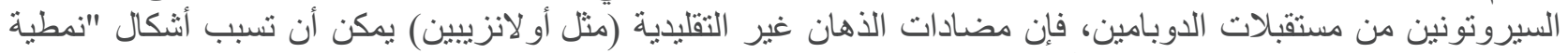

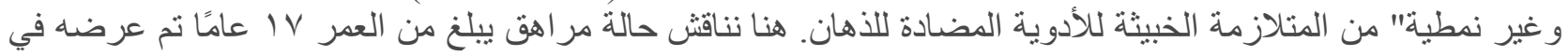

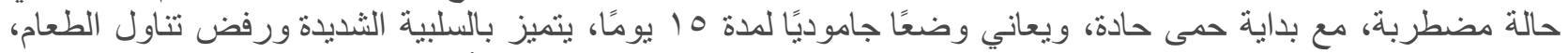

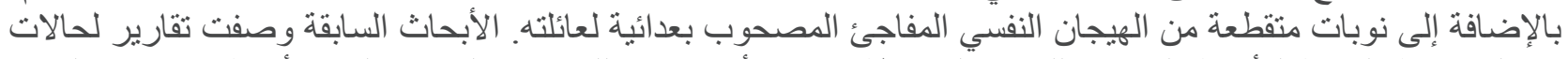

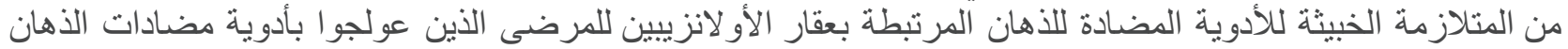

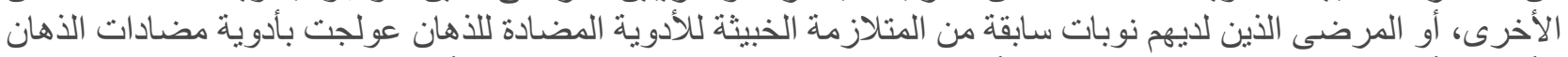

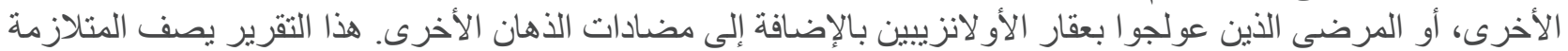

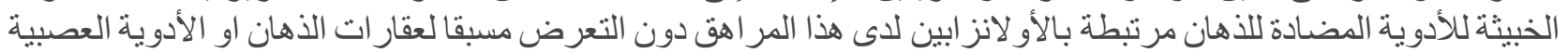
الاخرى. 